\title{
Operation and stability of antiguided flared amplifiers
}

\author{
S. Ramanujan and H. G. Winful \\ Department of Electrical Engineering, University of Michigan, 1301 Beal Avenue, Ann Arbor, \\ Michigan 48109
}

(Received 7 November 1995; accepted for publication 23 February 1996)

\begin{abstract}
We present a propagation model for the dynamics of antiguided flared amplifiers. This model takes into account diffraction, carrier diffusion, spatial hole burning, carrier-induced antiguiding, and spontaneous emission. Numerical results demonstrate that flared antiguides are significantly less susceptible to noise induced filamentation than broad area devices. (c) 1996 American Institute of Physics. [S0003-6951(96)02018-9]
\end{abstract}

Research in the area of compact high power diffraction limited sources for optical communications has produced several innovative and efficient devices. Of these devices, the antiguided laser array has demonstrated $1 \mathrm{~W}$ of power in a diffraction-limited beam. ${ }^{1}$ Another promising device is the master oscillator power amplifier using the broad-area flared amplifier. This device can produce in excess of $1 \mathrm{~W}$ of power. ${ }^{2}$ However, filamentation in the broad-area flared amplifier reduces the potential efficiency of the device. ${ }^{3-9} \mathrm{Re}-$ cent efforts by Botez et al. ${ }^{10}$ produced a device that incorporated the advantages of the flared amplifier design with lateral mode control of the antiguided laser. ${ }^{11,12}$ The result, an antiguided flared amplifier, can produce power comparable to that of the flared amplifier, yet is less prone to the filamentation that can affect broad area devices. We present in this letter an analysis of the antiguided flared amplifier that includes diffraction, diffusion, carrier-induced antiguiding, gain compression, and spontaneous emission. This analysis confirms that the antiguided flared amplifier is less prone to lateral mode distortion from filamentation and hence presents a viable option in the list of possible high power compact sources.

We begin by discussing the broad-area flared amplifier. The tapered amplifier receives an injected field that we assume to be Gaussian. The field propagates along the length of the amplifier under a contact whose shape is determined by the diffraction angle of the beam. This design offers several advantages over the traditional wide stripe or broad area amplifier. Because gain is only provided along the path of the defined lateral mode, the growth of alternate lateral modes is inhibited. Also, because the taper provides a gain region that is not symmetric in the direction of propagation, self-oscillation and feedback effects are reduced. However, several studies of tapered amplifiers have shown that noise either present on the input field or present in the amplifier can induce filamentation in the cw output of the amplifier. ${ }^{6,9}$ In the case of amplitude fluctuations, nonuniformities in the input field can lead to uneven saturation of the gain and promote lateral mode instabilities. ${ }^{6,7}$ Furthermore, noise within the amplifier can couple to the injected field through carrier induced antiguiding. ${ }^{9}$ The result is amplitude fluctuations that are further accentuated by the nonuniform saturation of the gain. The lateral field at the output facet of the amplifier exhibits distortion as does the associated phase. We see an example of a calculated cw output of the near-field with and without phase noise in Fig. 1.
The filamentation shown in Fig. 1 has a period of 10 $\mu \mathrm{m}$. This type of filamentation arises from the broad area nature of the device. An index-guided array structure will be less prone to such an instability because the gain profile is determined by the spacing of the arrays and not purely by the gain saturation. However, for index guided arrays, the gain region is reduced and, as a result, the amplification cannot approach that offered by the broad area devices. Also, elements in the array need not couple in-phase, and the far-field may be twin lobed.

Antiguided arrays offer certain advantages not seen in typical index-guided arrays. First, because the device depends on laterally propagating or radiative wave coupling as opposed to evanescent wave coupling, the resulting output power is significantly greater than that of index-guided arrays. Second, the array can be designed to ensure in-phase behavior at the output by spacing the antiguide elements an odd integer number of half-wavelengths apart. Finally, because the phase front is relatively flat, there need not be any external optics to correct for quadratic curvature. By combining the tapered contact with an underlying antiguide array structure, we hope to combine the positive elements of both devices in a single high power device.

The device we choose to examine is a $980 \mathrm{~nm}$ wavelength flared amplifier of length $2000 \mu \mathrm{m}$, initial guide width of $4 \mu \mathrm{m}$, and antiguide element spacing of $1 \mu \mathrm{m}$. There are 41 antiguides in the array. The source of injection is the output of a three core resonant optical waveguide array laser. ${ }^{12}$ The three element injection is to ensure that the input

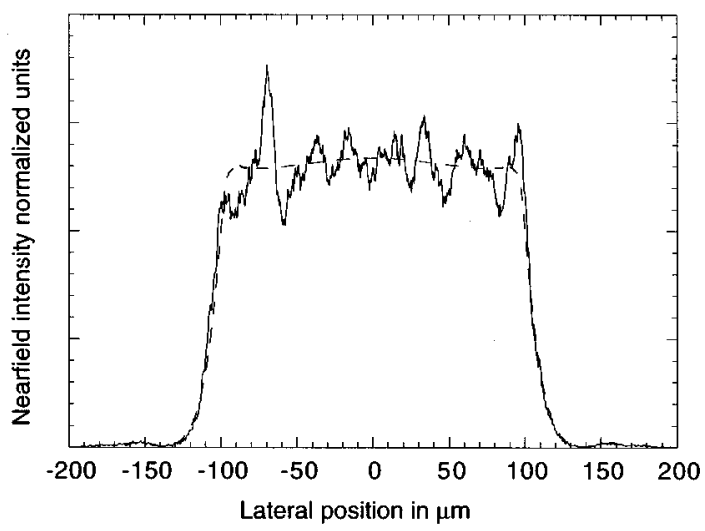

FIG. 1. Near-field for steady-state operation of the broad area flared amplifier. Solid line is near-field with noise and dashed line is near-field without noise. 
aperture of the amplifier is sufficiently filled. We also assume a linear interelement loss (equivalent to $50 \mathrm{~cm}^{-1}$ ) used in antiguide arrays to ensure modal discrimination.

The model employs a beam propagation model and solves field and carrier rate equations simultaneously. We assume an initial carrier density determined by the pump current at the input aperture of the device and propagate the input field along the length of the device. Variations to the carrier density are determined by solving the carrier rate equation. The direction of propagation is the $z$ direction, the lateral dimension is the $x$ direction, and the beam is assumed to be uniform in the $y$ direction. The unidirectional field equation in this coordinate space is ${ }^{13,14}$

$$
2 i k\left(\frac{1}{c} \frac{\partial E}{\partial t}+\frac{\partial E}{\partial z}\right)=\nabla_{\perp}^{2} E+k_{0}^{2} \Gamma \Delta \epsilon E+i k S(x, z, t) .
$$

In Eq. (1), $k$ is the propagation constant, $k_{0}$ is its value in free space, $z$ is the direction of propagation, $c$ is speed of light, $E$ is the field, $t$ is time, $\Gamma$ is the confinement factor in the $y$ direction, $\Delta \epsilon$ is the perturbation to the index of refraction, and $S(x, z, t)$ is the spontaneous emission term. The spontaneous emission noise term, $S(x, z, t)$, is ${ }^{13}$

$$
\begin{aligned}
S(x, z, t)= & A \sum_{j=0}^{J-1} \sum_{m=-M / 2+1}^{M / 2} \sum_{p=0}^{P-1}\{u[z-(j+1) \Delta z]-u \\
& (z-j \Delta z)\} \delta(t-p \Delta t) \exp \left[i\left(2 \pi m x / L_{x}+\phi_{j}^{m}\right)\right] .
\end{aligned}
$$

In Eq. (2), $A$ is the normalization constant, $M$ refers to the total number of grid points in the $x$ direction, $L_{x}$ is the width of the lateral window, the function $u(z)$ is the unit step function, and $\phi_{j}^{m}$ is the two-dimensional random phase. The spontaneous emission sources are uniformly distributed contributions over $2 \pi$ for each $\Delta z$. This method provides random numbers distributed in both the $x$ and the $z$ dimensions. The normalization constant is determined from the photon rate equation for this device. The photon rate equation is ${ }^{15}$

$$
\dot{P}=\left(G_{m}-\gamma_{m}\right) P_{m}+\beta_{s p} C_{1} N^{2} .
$$

In Eq. (3), $P$ is the photon density, $G_{m}$ is the modal gain, $\gamma_{m}$ is the modal loss, $C_{1}$ is the radiative recombination coefficient, and $N$ is the carrier density. The constant $\beta_{s p}$ has been empirically determined to be between $10^{-4}$ and $10^{-5}$ for index guided devices ${ }^{15}$ in similar material. We then estimate the value of the constant $A$ from $A=\sqrt{\left(R_{s p} \Delta t / \Delta z\right)}$. The next term in (1) is the perturbation to the index of refraction given by: ${ }^{14}$

$$
\begin{aligned}
\Delta \epsilon= & 2 n_{a} \Delta n_{\mathrm{eff}}-\frac{a n_{a}(\alpha+i)}{k_{0}\left(1+\sigma|E|^{2}\right)}\left(N-N_{0}\right) \\
& +\frac{i n_{c}(1-\Gamma)}{k_{0} \Gamma} \alpha_{c} .
\end{aligned}
$$

In (4), $n_{a}$ is the active region index, $\Delta n_{\text {eff }}$ is the built in refractive index difference in the lateral direction, $a$ is the gain cross section, $\alpha$ is the antiguiding parameter, $\sigma$ is the gain compression term, $N_{0}$ is the carrier density required for transparency, $n_{c}$ is the cladding index, and $\alpha_{c}$ is the cladding loss.
TABLE I. Antiguide parameter values.

\begin{tabular}{lcc}
\hline \hline \multicolumn{1}{c}{ Parameter } & Symbol & Value \\
\hline Index step & $\Delta n$ & -0.01 \\
Cladding loss & $\alpha_{c}$ & $0.0005 / \mu \mathrm{m}$ \\
Antiguiding & $\alpha$ & 3 \\
Transparency density & $N_{0}$ & $1.1 \times 10^{6} / \mu \mathrm{m}^{3}$ \\
Diffusion length & $l_{d}$ & $3 \mu \mathrm{m}$ \\
Differential gain & $a$ & $1.3 \times 10^{-8} \mu \mathrm{m}^{2}$ \\
Cladding index & $n_{c}$ & 3.3 \\
Active region index & $n_{a}$ & 3.4 \\
Effective index & $n_{e}$ & 3.34 \\
Nonradiative & $C_{0}$ & $1 \times 10^{8} / \mathrm{s}$ \\
Recombination & & \\
Radiative recombination & $C_{1}$ & $1.25 \times 10^{-10} \mu \mathrm{m}{ }^{3} / \mathrm{ps}$ \\
Auger recombination & $C_{2}$ & $3 \times 10^{-29} \mathrm{~cm} / \mathrm{s}$ \\
Wavelength & $\lambda$ & $980 \mathrm{~nm}$ \\
Current spreading & $l_{s}$ & $3 \mu \mathrm{m}$ \\
Stripe width & $w_{s}$ & $4 \mu \mathrm{m}$ \\
Center to center width & $w_{c}$ & $4.925 \mu \mathrm{m}$ \\
Confinement factor & $\Gamma$ & 0.02 \\
\hline \hline
\end{tabular}

The carrier density is determined from ${ }^{14}$

$$
\frac{\partial N}{\partial t}=j(x)-\frac{N}{\tau_{s}}-\frac{G_{m}\left(N-N_{0}\right)}{1+\sigma|E|^{2}}|E|^{2}+D_{e} \frac{\partial^{2} N}{\partial x^{2}} .
$$

The pump current, $j(x)$ is uniform over the tapered guide region and decays exponentially at the edges of the contact. The modal gain $G_{m}$ is given by $a \Gamma v_{g}$, where $v_{g}$ is the group velocity. The final term in (5) is diffusion. The values used in evaluation of all the given equations are shown in Table I. ${ }^{14,15}$

We can approximate the turn-on transient from temporal solutions of the equations. We assume an initial carrier density throughout the amplifier given by the pump current. We then propagate the input field along the length of the amplifier using the assumed carrier density and solve the carrier rate equation at each propagation step. The altered carrier density is stored and used for the next time step. Each subsequent time step is approximated by propagating the same initial field along the amplifier and using the carrier density determined from the previous field. In this manner we approximate the temporal change of the carrier density and the resulting changes in the field while explicitly including the time variation of the spontaneous emission. This method can be used to examine the steady-state results by simply allowing the simulation to reach stead state. We find that the results do not differ from simply solving for the steady-state solution. However, the transient solution can help determine the time scale on which filaments, when present, arise.

We begin by examining the state of the amplifier 25 ps after turn-on. Figure 2 is the near-field of the amplifier with phase noise. The result for simulations with and without noise are indistinguishable. Thus, we can conclude there is no noise-induced near-field filamentation. The near-field peaks correspond to the locations of the antiguide elements. This solution is stable in steady state, and demonstrates the advantages of an antiguided substructure. When the index step is closer to resonance, the near-field better fills the aperture and demonstrates better coupling. The simulation results presented so far, use interelement absorbers to control the growth of field in the interelement regions and hence 


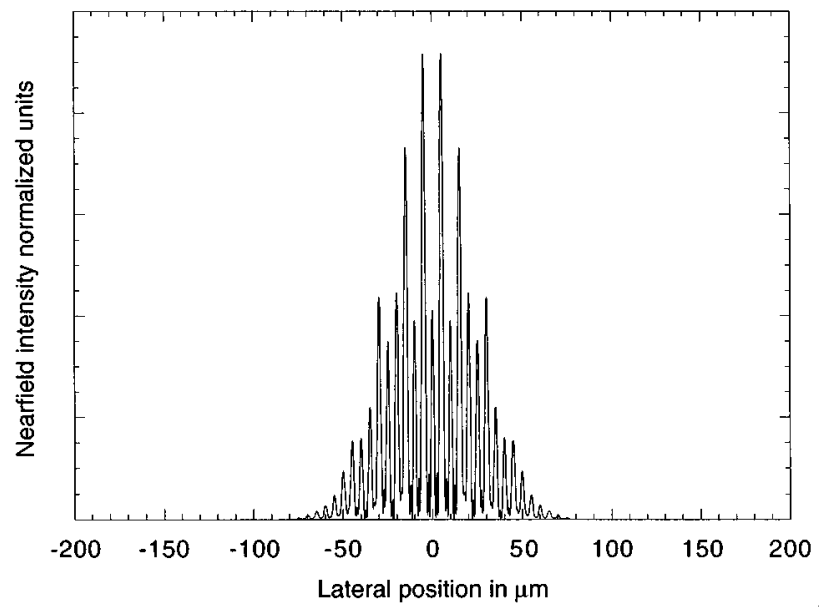

FIG. 2. Near-field 25 ps after turn-on for the flared antiguided amplifier.

control the lateral mode. A steady-state analysis shows that because the injected signal is stable, elimination of interelement loss only slightly corrupts the far-field as can be seen in Fig. 3. The power remains well-confined in the central lobe,

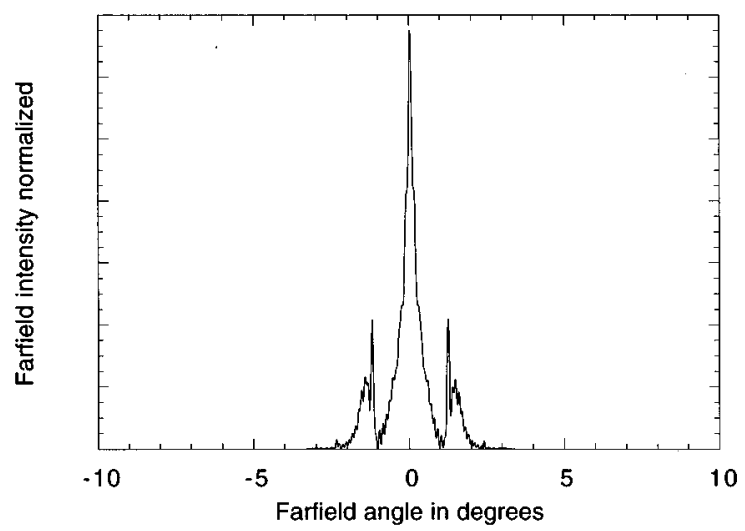

FIG. 3. Far-field for steady-state operation of the flared antiguided amplifier with no interelement loss. and as with the previous results, there is no noise-induced filamentation. By carefully tailoring the antiguide widths and spacing, the side lobes in the far-field can be reduced. ${ }^{1,12}$ As with the near-field, the far-field simulation results with and without noise are identical. Steady-state results also determine that amplification can approach 15 times the injected signal.

The antiguided flared amplifier shows great promise as a high power compact source. Combining the high gain region of the flared amplifier, with the lateral mode stability of the antiguided waveguide can reduce the problems associated with each independent device. Noise induced filamentation is eliminated and temporal stability is maintained.

${ }^{1}$ C. Zmudzinski, D. Botez, L. J. Mawst, C. Tu, and L. Frantz, Appl. Phys. Lett. 62, 2914 (1993).

${ }^{2}$ S. O'Brien, F. F. Welch, R. Parke, D. Mehuys, K. Dzurko, R. J. Lang, R. Waarts, and D. Sciferes, IEEE J. Quantum Electron. 29, 2052 (1993).

${ }^{3}$ R. J. Lang, D. Mehuys, A. Hardy, K. M. Dzurko, and D. Welch, Appl. Phys. Lett. 62, 1209 (1993).

${ }^{4}$ R. J. Lang, A. Hardy, R. Parke, D. Mehuys, S. O'Brien, J. Major, and D. Welch, IEEE J. Quantum Electron. 29, 2044 (1993).

${ }^{5}$ A. H. Paxton and G. C. Dente, J. Appl. Phys. 70, 2921 (1991).

${ }^{6}$ L. Goldberg, M. Surette, and D. Mehuys, Appl. Phys. Lett. 62, 2304 (1993).

${ }^{7}$ G. Dente and M. L. Tilton, IEEE J. Quantum Electron. 29, 76 (1993).

${ }^{8}$ R. J. Lang, D. Mehuys, D. Welch, and L. Goldberg, IEEE J. Quantum Electron. 30, 685 (1994).

${ }^{9}$ S. Ramanujan and H. G. Winful, IEEE J. Quantum Electron (to be published).

${ }^{10}$ D. Botez, M. Jansen, C. Zmudzinski, L. J. Mawst, P. Hayashida, and C. Tu, Appl. Phys. Lett. 63, 3113 (1993).

${ }^{11}$ C. Zmudzinski, D. Botez, L. Mawst, and R. Nabiev, Tech. Dig. CLEOEurope Conference, Amsterdam, The Netherlands, Sept. 1994, Paper CTuP3.

${ }^{12}$ C. Zmudzinski, D. Botez, L. J. Mawst, A. Bhattacharya, M. Nesnidal, and R. F. Nabiev, IEEE J. Select Topics Quantum Electron. 1, 129 (1995).

${ }^{13}$ M. D. Feit and J. A. Fleck, J. Opt. Soc. Am. B 7, 2048 (1990).

${ }^{14}$ L. Rahman and H. G. Winful, IEEE J. Quantum Electron. 30, 1405 (1994).

${ }^{15}$ G. P. Agrawal and N. K. Dutta, Semiconductor Lasers (Van Nostrand, New York, 1993).

${ }^{16}$ S. C. Chapra and R. P. Canale, Numerical Methods for Engineers (McGraw-Hill, New York, 1988).

${ }^{17}$ G. R. Hadley, Opt. Lett. 16, 624 (1991). 